Franco CAPORALE, Mirco GUIDI

\title{
Azanus ubaldus (Stoll, 1782) nuovo per l'Italia (Lepidoptera Lycaenidae)
}

Riassunto: Azanus ubaldus (Stoll, 1782) è segnalato per la prima volta in Italia su esemplari raccolti nell'isola di Lampedusa.

\begin{abstract}
Azanus ubaldus (Stoll, 1782), new to Italy (Lepidoptera Lycaenidae).
Azanus ubaldus Stoll, 1782 is reported for the first time in Italy on the basis of some specimens collected in Lampedusa island (Sicily).
\end{abstract}

Key words: Lycaenidae, Azanus, Italy, Lampedusa island.

Il genere Azanus Moore, 1881 (Lepidoptera Lycaenidae Polyommatinae) essenzialmente africano, comprende una decina scarsa di specie, tutte infeudate su piante del genere Acacia Miller e solo saltuariamente su altre Fabaceae Lindl (D'Abrera, 2009).

Il genere Azanus Moore, 1881 si differenzia dai vicini generi Tarucus Moore, 1881 e Leptotes Scudder, 1876 (=Syntarucus Butler, 1900) per avere la prima nervatura radiale delle ali anteriori completamente anastomizzata con la subcostale, mentre in Leptotes essa è completamente libera ed in Tarucus solo strettamente addossata (Verity, 1943).

Delle 10 specie appartenenti al genere Azanus due sono endemiche del Madagascar, un'altra è diffusa lungo la fascia delle foreste equatoriali africane (Larsen, 2005). Tutte le altre specie risultano distribuite nelle parti aride dall'Africa tropicale meridionale ed orientale ai paesi mediterranei; tra queste ultime Azanus jesous Guerin, 1847 ed Azanus ubaldus (Stoll, 1782) sono presenti anche nel continente asiatico, dall'India allo Sri Lanka, all'Arabia Saudita e a diversi paesi del medio oriente (Seitz, 1911).

Azanus ubaldus in particolare è diffuso dalle zone tropicali aride dell'Africa meridionale alla Somalia nell'Africa orientale (D'Abrera, 2009), questo dato contrasta con quanto affermato da Larsen (2005) che non conosce segnalazioni al disotto dei $10^{\circ}$ di latitudine Nord, mentre cita come zona di elezione del taxon i paesi sub-sahariani del Sahel.

Ad occidente la sua distribuzione attraverso il Senegal raggiunge il Marocco e le isole Canarie; sulle coste mediterranee è presente in Algeria, Tunisia ed Egitto e, nel Vicino Oriente, in Israele e Giordania (Tolman \& Lewington, 1997).

Le specie appartenenti al genere Azanus pre- sentano caratteri morfologici abbastanza omogenei: capo piccolo con palpi ben sviluppati; antenne sottili, lunghe circa quanto la metà del bordo costale con clava appiattita di dimensioni ridotte. Torace robusto con zampe esili, addome lungo e sottile. Ali ben sviluppate, le anteriori allungate presentano apice acuto e margine costale leggermente convesso; l'angolo anale delle posteriori, leggermente ottuso, lascia supporre la presenza di codette rudimentali (Seitz, 1911).

Nonostante le dimensioni molto ridotte le specie del genere Azanus sono capaci di volo rapido e sicuro, che conferma il comportamento migratorio di alcune di esse (Tolman \& Lewington, 1997).

La biologia larvale ed il voltinismo non sono ad oggi completamente conosciuti, ma pare sicuro che le larve, vengano accudite da formiche dei generi Camponotus Mayr e Prenolepis Mayr (Tolman \& Lewington, 1997).

I genitali della specie sono descritti da Stempffer (1967).

Acronimi utilizzati: $\mathrm{FC}=$ collezione Caporale (Bologna); $\mathrm{MG}=$ collezione Guidi (Bubano BO).

\section{Azanus ubaldus (Stoll, 1782)}

Materiale esaminato. Sicilia, Lampedusa (Agrigento) dintorni del campo sportivo, in zona fortemente antropizzata, al livello del mare, 26.IX.2010 1 今, M. Guidi legit (MG) posato a riscaldarsi al sole su uno stelo secco di Foeniculum vulgare Miller (Pignatti 1982). Sicilia, Lampedusa (Agrigento) Albero Sole $133 \mathrm{~m}$ s.l.m, massimo rilievo dell'isola 27.IX.2010 10

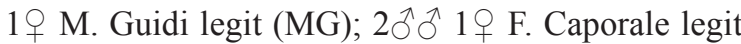
(FC) durante le brevi schiarite di una giornata nuvolosa, e a tratti piovosa, mentre volavano bottinando sulla fioritura tardiva di alcuni individui di Acacia 
karoo Hayne (Pignatti 1982). Acacia karoo sull'isola rappresenta probabilmente la principale pianta alimentare del taxon. Si tratta di una specie originaria dell'Africa meridionale, che viene coltivata in Sicilia e sulle isole minori per formare siepi impenetrabili e per il consolidamento del suolo (Pignatti 1982).

I pochi esemplari di A. ubaldus raccolti, probabilmente appartenenti tutti all'ultima generazione dell'anno 2010, pur confermando le ridotte dimensioni del taxon, denotano una certa variabilità in quanto i $4 \hat{\partial} \widehat{\partial}$ presentano una lunghezza dell'ala anteriore variabile tra gli 8 e i $10.75 \mathrm{~mm}$ mentre nelle 2 우, apparentemente più omogenee, questa misura rispettivamente 9.50 e $10 \mathrm{~mm}$.

La pagina superiore delle ali maschili presenta una colorazione azzurro pallida con frange bianche e bordi bruno nerastri ben delimitati; è presente al centro dell'ala anteriore una macchia androconiale più scura formata da squame strette e di lunghezza variabile; sull'ala posteriore sono ben visibili in prossimità del margine anale due macchie tondeggianti nere.

La pagina superiore delle ali femminili, negli esemplari raccolti dagli autori, appare abbastanza uniformemente bruna e per lo più presenta un inscurimento basale. Le macchie nere prossime al margine

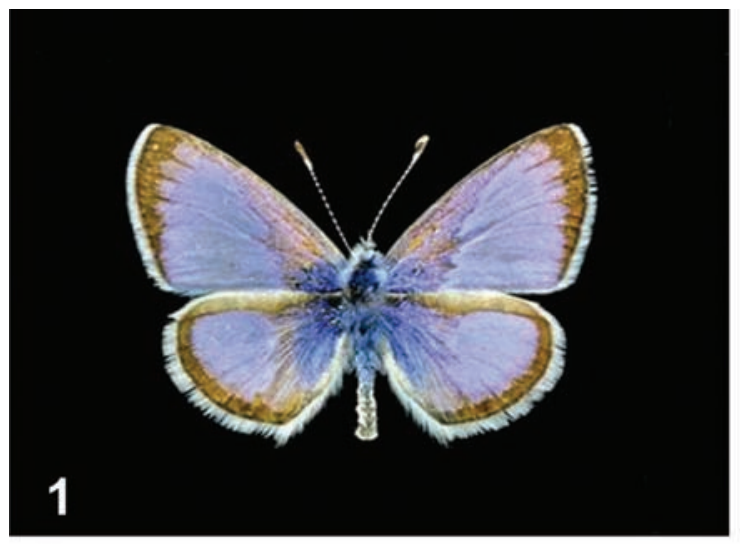

$10 \mathrm{~mm}$ anale sono più nitide nella femmina che nel maschio.

La pagina inferiore delle ali maschili presenta una colorazione bruno-grigiastra chiara, attraversata da striature biancastre subparallele ondulate e discontinue. La striatura premarginale comprende una serie di lunule abbastanza regolari che termina nell'angolo anale con le due macchie nere visibili anche sulla pagina superiore. Oltre a questa coppia di macchie nell'ala posteriore ne sono presenti altre quattro al margine dell'area basale e una lungo la nervatura costale, al limite dell'area discale, non visibili sulla pagina superiore.

Nella femmina la pagina inferiore appare in tutto simile a quella del maschio, ma la sua colorazione di base brunastra e le macchiette nere, normalmente più sviluppate, la rendono più contrastata (Figg. 1-4).

Gli esemplari raccolti sono depositati nelle collezioni degli autori.

Scopo di ulteriori ricerche sarà di accertare che la presenza del taxon sull'isola sia stabile e non frutto di casuali migrazioni dalle antistanti, e relativamente vicine, coste mediterranee dei paesi Nord-africani, definendone nel contempo il voltinismo nel nostro ambiente. Si dovrà inoltre stabilire con sicurezza la pianta, o le piante, nutrici, utilizzate sull'isola italiana.

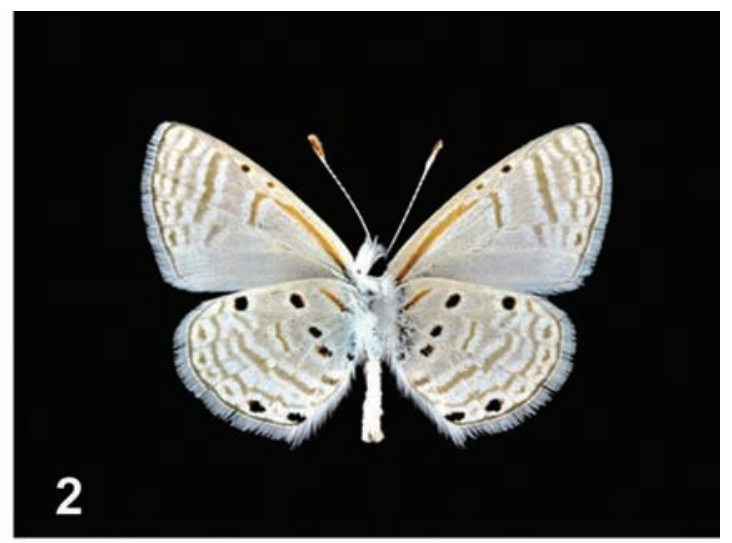

Figg. 1-2. Azanus ubaldus (Stoll) §̊; Lampedusa (AG): Campo sportivo 1-habitus dorsale 2- habitus ventrale. (Foto G. Fiumi). 

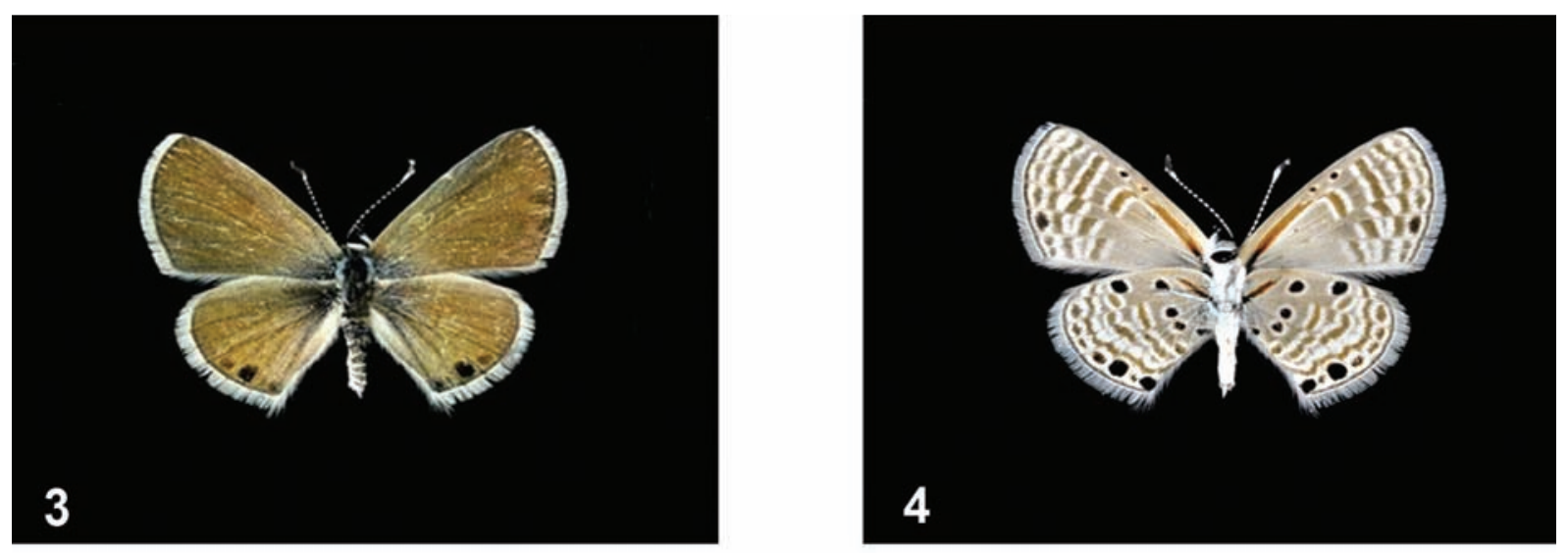

$10 \mathrm{~mm}$

Figg. 3-4. Azanus ubaldus (Stoll) $q$; Lampedusa (AG): Albero Sole 3-habitus dorsale 4- habitus ventrale. (Foto G. Fiumi).

\section{BIBLIOGRAFIA}

D’Abrera B., 2009 - Butterflies of the Afrotropical Region. Hill House Publishers, Part III: Lycaenidae, Rhiodinidae: 816-817. LARSEN T.B., 2005 - Butterflies of West Africa. Apollo Books: 267-268.

PignATti S., 1982 - Flora d'Italia. Edagricole, Bologna, 2: 205. 1: 626.

Seitz A., 1911 - Lés Macrolepidopteres du Globe. Cabinet Entomologique E. Le Moult, Paris, 1: 293-294.

STEMPFFER H., 1967 - The genera of the African Lycaenidae (Lepidoptera: Rhopalocera). Bulletin of he British Museum, Natural History, (Entomology), Supplement 10: 1-322.

Tolman T. \& Lewington R.,1997 - Collins Field Guide Butterflies of Britain \& Europe Harper Collins: 85.

VERITY R., 1943 - Le farfalle diurne d'Italia. Marzocco S.A. Firenze, 2: 79.

Indirizzo degli autori:

Franco Caporale, via Mezzofanti 3, 40137 Bologna, Italia. E-mail: fbcaporale@gmail.com

Mirco Guidi, via Amendola 18/A, 40020 Bubano, Imola (BO), Italia. 